\title{
Measurements of Cosmic Magnetism with LOFAR and SKA
}

\author{
R. Beck \\ Max-Planck-Institut für Radioastronomie, Auf dem Hügel 69, 53121 Bonn, Germany
}

\begin{abstract}
The origin of magnetic fields in stars, galaxies and clusters is an open problem in astrophysics. The nextgeneration radio telescopes Low Frequency Array (LOFAR) and Square Kilometre Array (SKA) will revolutionize the study of cosmic magnetism. "The origin and evolution of cosmic magnetism" is a key science project for SKA. The planned all-sky survey of Faraday rotation measures (RM) at $1.4 \mathrm{GHz}$ will be used to model the structure and strength of the magnetic fields in the intergalactic medium, the interstellar medium of intervening galaxies, and in the Milky Way. A complementary survey of selected regions at around $200 \mathrm{MHz}$ is planned as a key project for LOFAR. Spectropolarimetry applied to the large number of spectral channels available for LOFAR and SKA will allow to separate RM components from distinct foreground and background regions and to perform 3-D Faraday tomography of the interstellar medium of the Milky Way and nearby galaxies. Deep polarization mapping with LOFAR and SKA will open a new era also in the observation of synchrotron emission from magnetic fields. LOFAR's sensitivity will allow to map the structure of weak, extended magnetic fields in the halos of galaxies, in galaxy clusters, and possibly in the intergalactic medium. Polarization observations with SKA at higher frequencies $(1-10 \mathrm{GHz})$ will show the detailed magnetic field structure within the disks and central regions of galaxies, with much higher angular resolution than presentday radio telescopes.
\end{abstract}

\section{Introduction}

Understanding the Universe is impossible without understanding magnetic fields. They fill interstellar and intracluster space, affect the evolution of galaxies and galaxy clusters,

Correspondence to: R. Beck

(rbeck@mpifr-bonn.mpg.de) contribute significantly to the total pressure of interstellar gas, are essential for the onset of star formation, and control the density and distribution of cosmic rays in the interstellar medium (ISM) and in the intracluster medium (ICM). In spite of their importance, the evolution, structure and origin of magnetic fields are all still open problems in fundamental physics and astrophysics. When and how were the first magnetic fields in the Universe generated? Was there is a connection between magnetic field formation and structure formation in the early Universe? Were the fields in young galaxies and clusters primordial or generated in the galaxies themselves? How did magnetic fields evolve as galaxies evolve? What are the strength and structure of the magnetic field of the intergalactic medium (IGM)?

Most of what we know about astrophysical magnetic fields comes through the detection of radio waves. Synchrotron emission measures the total field strength, while its polarization yields the orientation of the regular field in the sky plane and also gives the field's degree of ordering (Figs. 4-6). Faraday rotation of the polarization vector when the wave passes through magnetized plasma gives a measure of the regular field along the line of sight (Fig. 2). The combination yields a three-dimensional view of the regular field. The Zeeman effect provides an independent measure of field strength in cold gas clouds. However, measuring astrophysical magnetic fields is a difficult topic, restricted to nearby or bright objects when observing with present-day radio telescopes.

\section{LOFAR}

The Low Frequency Array (LOFAR) is a new-generation phased array radio telescope which will observe in the frequency bands $30-80 \mathrm{MHz}$ and $110-240 \mathrm{MHz}$. It is under construction in the Netherlands (see www.lofar.org and www.lofar.de). The first international station is under con-

Published by Copernicus Publications on behalf of the URSI Landesausschuss in der Bundesrepublik Deutschland e.V. 


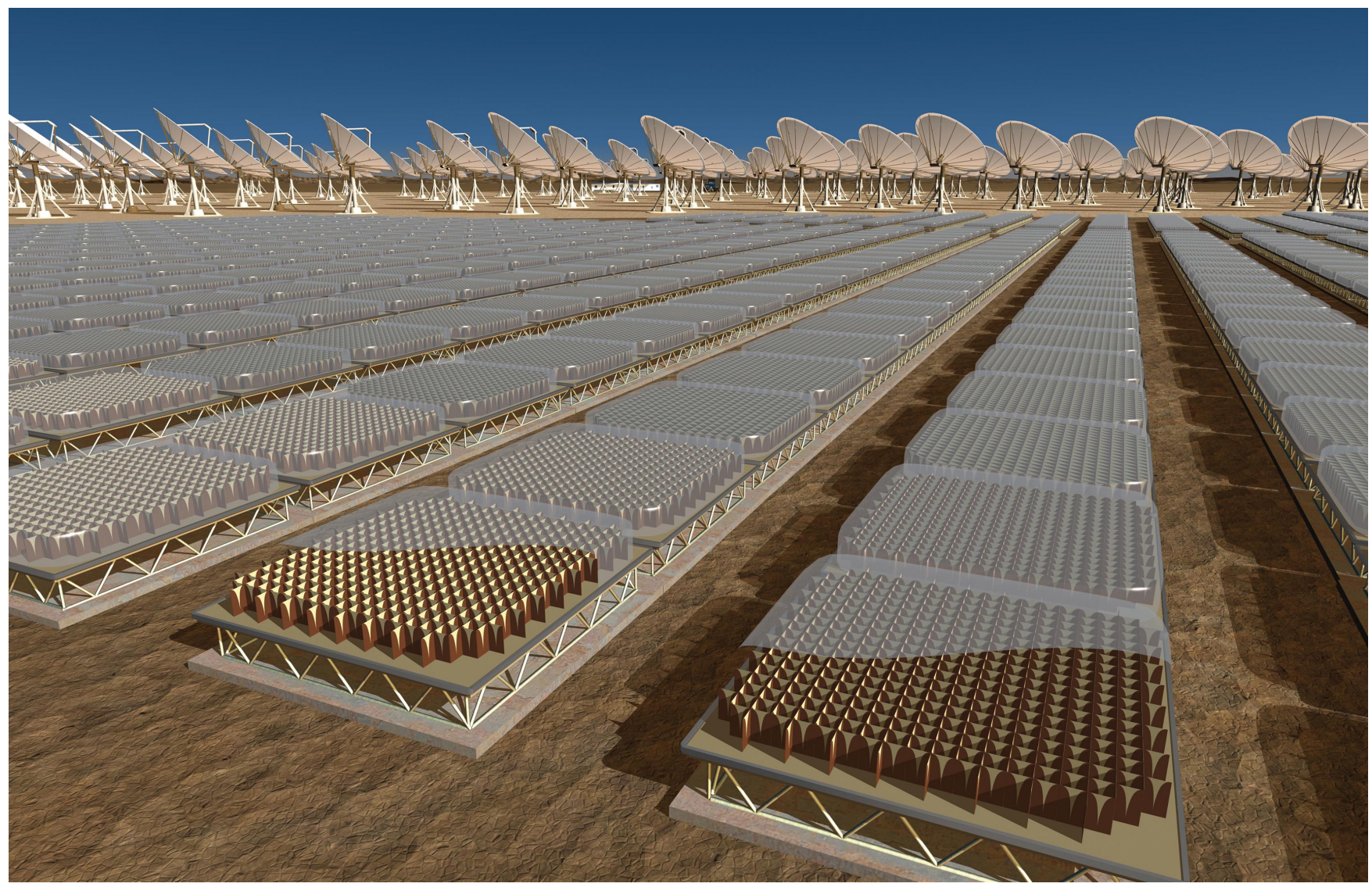

Fig. 1. SKA reference design (SKA Project Office and XILOSTUDIOS).

struction next to the Effelsberg 100-m telescope (Reich, this volume).

Low-frequency radio emission traces low-energy cosmicray electrons which suffer less from energy losses and hence can propagate further away from their sources into regions with weak magnetic fields. The lifetime of electrons in galaxies is generally limited by synchrotron losses and increases with decreasing frequency and decreasing field strength. In a $5 \mu \mathrm{G}$ field the lifetime of electrons emitting in the LOFAR bands is $(2-5) \cdot 10^{8} \mathrm{yr}$. In magnetic fields weaker than $3.25 \mu \mathrm{G} \cdot(z+1)^{2}$ (where $z$ is the redshift) the electron lifetime is limited by the Inverse Compton effect with the photons of the cosmic micowave background (CMB), so that the lifetime of electrons observed at frequency $v$ decreases weakly with decreasing field strength. Electrons with maximum lifetimes of $\sim 10^{9} \mathrm{yr}$ can be observed at $50 \mathrm{MHz}$ in fields of about $3 \mu \mathrm{G}$ strength. Travelling through the hot gas in galactic halos or clusters with the Alfvén speed, they can propagate more than $100 \mathrm{kpc}$ from their places of origin.

LOFAR will give access to the so far totally unexplored domain of weak magnetic field strengths. For a fixed signalto-noise ratio of polarized intensity, the minimum detectable strength of the regular field is proportional to $v^{2}$. Hence, ob- serving diffuse polarized emission at low frequencies with LOFAR will reveal objects with weak fields such as galaxy halos and galaxy clusters. Faraday rotation increases with $v^{-2}$, so that LOFAR will be able measure very small rotation measures (RM) and detect weak magnetic fields and low electron densities which are unobservable with present-day telescopes and even at the higher frequencies of SKA. Faraday rotation of polarized background sources with LOFAR may even allow to detect magnetic fields in the intergalactic medium (Sect. 9).

Four key science projects lead by Dutch scientists are under development on the following topics: The epoch of reionization, extragalactic surveys, transients and pulsars, and cosmic rays. The German Long Wavelength Consortium (GLOW) plans to perform further key projects on solar radio astronomy, jets, and cosmic magnetism.

\section{SKA}

The Square Kilometre Array (SKA) is an international project for the next decade to build a huge radio telescope with a collecting area of about one square kilometre, dis- 


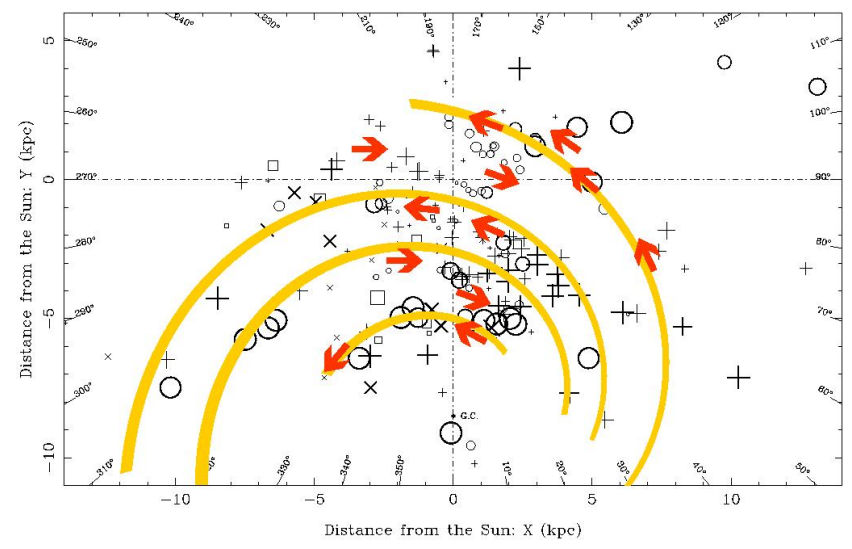

Fig. 2. Bird's eye view of the distribution of the Faraday rotation measures (RM) of pulsars within $8^{\circ}$ of the Galactic plane. Positive RMs are shown as crosses and X, negative RMs as circles and open squares. The symbol sizes are proportional to the square root of $|R M|$, with the limits of 5 and $250 \mathrm{rad} / \mathrm{m}^{2}$. The directions of a field model are given as arrows. The approximate location of four optical spiral arms is indicated as dotted lines (from Han et al., 1999).

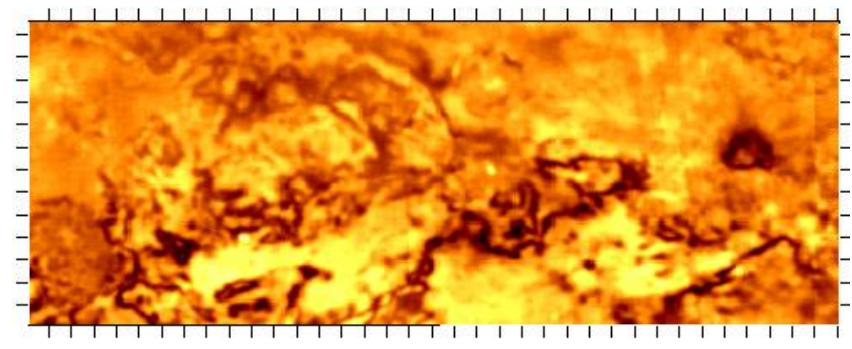

Fig. 3. Polarized intensity around the plane of the Milky Way $(l=$ $\left.150^{\circ}-174^{\circ}, b=-4.5^{\circ}-+4.5^{\circ}\right)$ at $1.4 \mathrm{GHz}$, combined from data of the Effelsberg and Dwingeloo radio telecopes (from Reich et al., 2004).

tributed over a large number of small antennas concentrated in several stations (Fig. 1). Requirements for the SKA design include a full frequency coverage of $0.3-20 \mathrm{GHz}$ and a field of view at $1.4 \mathrm{GHz}$ of at least $1 \mathrm{deg}^{2}$ which can be fully imaged at $1^{\prime \prime}$ resolution. The frequency range $0.3-1 \mathrm{GHz}$ will be covered by phased arrays, based on the experience with LOFAR and SKA prototypes, while classical parabolic dishes are considered at higher frequencies. A significant fraction of the collecting area will be concentrated into the central core of diameter $\sim 5 \mathrm{~km}$, and longest baselines of $\sim 3000 \mathrm{~km}$ are planned. The site selection process has identified South Africa and Western Australia as the best sites for the SKA core and inner stations, with extensions to West and East Africa and to New Zealand, respectively. Construction of Phase 1 with $10 \%$ collecting area is planned for 2012, construction of the full array for 2015 . SKA prototypes are un-

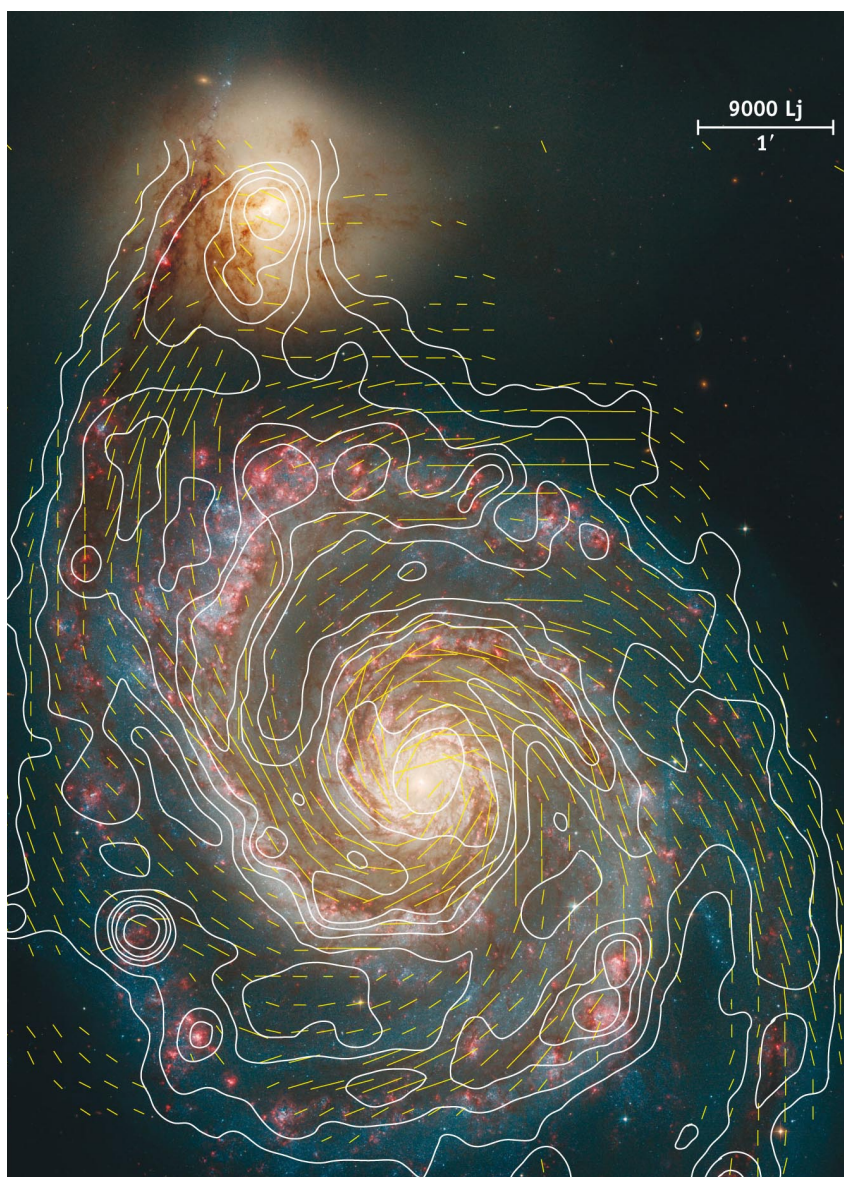

Fig. 4. Total radio emission (contours) and magnetic field vectors at $4.8 \mathrm{GHz}$, combined from observations with the VLA and the Effelsberg telescope. The underlying image is from the Hubble Space Telescope (A. Fletcher and R. Beck / Hubble Heritage Team, graphics by the magazine "Sterne und Weltraum").

der construction in several countries. The European Community has started the SKA Design Study (SKADS) programme which involves 29 institutes from 12 countries.

Six SKA key science projects have been selected on the followings topics: The Dark Ages, galaxy evolution and large-scale structures, testing theories of gravitation, the Cradle of Life, cosmic magnetism, and exploration of the unknown. A science case book was published in 2004 (Carilli and Rawlings, 2004).

For more information see www.skatelescope.org.

\section{Faraday rotation and spectro-polarimetry}

Much of what LOFAR and SKA can contribute to our understanding of magnetic fields will come from their polarimetric capabilities. The crucial specifications are high polarization purity and multichannel spectro-polarimetric capability. 
The former will allow detection of the relatively low linearly polarized fractions $(\leq 1 \%)$ from most astrophysical sources, while the latter will enable accurate measurements of Faraday rotation measures (RMs), intrinsic polarization position angles and Zeeman splitting.

With current instruments, the only way to simultaneously determine accurate values for both the RM and intrinsic polarization position angle is to make several observations at various frequencies across a broad frequency range. This is not only time-consuming, but the analysis must proceed cautiously, since various depolarizing effects have a strong frequency dependence (Sokoloff et al., 1998).

The high sensitivity and broad bandwidth of LOFAR and SKA eliminate these difficulties: a single spectropolarimetric observation at a single IF can simultaneously provide good estimates of both RM and position angle, the limiting factor being the accuracy of ionospheric corrections to the observed Faraday rotation. For example, at an observing frequency of $1.4 \mathrm{GHz}$ with a fractional bandwidth of $25 \%$, a 1-min SKA observation of a source with a linearly polarized surface brightness of $\sim 8 \mu \mathrm{Jy}$ beam $^{-1}$ will yield a $\mathrm{RM}$ determined to an accuracy $\Delta \mathrm{RM} \approx \pm 5 \mathrm{rad} \mathrm{m}^{-2}$, sufficient to measure a regular field of $5 \mu \mathrm{G}$ strength and $100 \mathrm{pc}$ correlation length in a plasma of $0.05 \mathrm{~cm}^{-3}$ electron density.

Making use of its wide-band polarization facility, LOFAR can detect even smaller rotation measures, possibly down to about $0.1 \mathrm{rad} \mathrm{m}^{-2}$ (giving $36^{\circ}$ total rotation at $120 \mathrm{MHz}$ and $13^{\circ}$ at $200 \mathrm{MHz}$ ), and hence will become the telescope to measure the weakest cosmic magnetic fields so far.

In the relatively strong magnetic fields of the interstellar medium in the Milky Way and other galaxies, low-frequency polarization is generally low due to various depolarization effects (Sokoloff et al., 1998). The method of RM synthesis, based on multichannel spectro-polarimetry, allows to measure a large range of RM values and to separate RM components from distinct regions along the line of sight (Brentjens and de Bruyn, 2005). This can be used for Faraday tomography of the interstellar medium in the Milky Way and in the disks and central regions of nearby galaxies.

\section{Rotation measure surveys}

Currently $\sim 1800$ extragalactic sources and $\sim 300$ pulsars have measured RM data (Kronberg, 1994; Brown et al., 2003; Han et al., 2006). These have proved useful probes of magnetic fields in the Milky Way, in nearby galaxies, and in clusters. However, the sampling of such measurements over the sky is very sparse.

With its low frequency range and wide-band polarization facility, LOFAR can detect weak rotation measures possibly down to about $0.1 \mathrm{rad} \mathrm{m}^{-2}$ (Sect. 4). An RM survey of selected fields around $200 \mathrm{MHz}$ is the ideal project to measure weak magnetic fields and to develop the analysis tools needed for SKA. However, the RM contribution from the
Galactic foreground in the Galactic plane are much larger than the RMs from galaxy halos, clusters, and the IGM. Application of RM synthesis (Sect. 4) with sufficiently large RM range and RM resolution will be essential to separate the RM components along the line of sight.

The key platform on which to base the SKA's studies of cosmic magnetism will be the all-sky RM survey at $1.4 \mathrm{GHz}$, in which spectro-polarimetric continuum imaging of $10000 \mathrm{deg}^{2}$ of the sky can yield RMs for approximately $2 \times 10^{4}$ pulsars and $2 \times 10^{7}$ compact polarized extragalactic sources (see Gaensler et al., 2004 for a detailed description). This data set will provide a grid of RMs at a mean spacing of $\sim 30^{\prime}$ between pulsars and just $\sim 90^{\prime \prime}$ between extragalactic sources.

The structure of the magnetic field in our Milky Way can be determined with help of pulsar RMs. The analysis of current data indicates a "bisymmetric" field with several field reversals (Fig. 2, Han et al., 1996, 2006). However, the uncertainties are still large. The large sample of pulsar RMs obtained with the SKA, combined with distance estimates to these sources from parallax or from their dispersion measures, can be inverted to yield a complete delineation of the magnetic field in the spiral arms and disk on scales $\geq 100 \mathrm{pc}$ (Stepanov et al., 2002). Furthermore, the magnetic field geometry in the Galactic halo and the outer parts of the disk can be studied using the all-sky RM grid.

With the sensitivity of the SKA, deep observations of nearby galaxies and galaxy clusters can provide a huge number of background RMs and thus allow detailed maps of the magnetic structure. A large sample of objects could be studied in this way.

\section{Polarization mapping of cosmic magnetic fields}

The unprecedented sensitivity of LOFAR and SKA will allow to map the polarized synchrotron emission to much fainter levels and/or with much better resolution than with present-day telescopes. While LOFAR will concentrate on the weak, diffuse emission from galaxy halos and the intracluster medium of galaxy clusters, SKA will trace detailed field structures in the Milky Way and in galaxies.

Small-scale structures and turbulence in the interstellar medium of the Milky Way can be probed using RM synthesis (Sect. 4). Foreground ionized gas generates frequencydependent Faraday features when viewed against diffuse Galactic polarized radio emission (Fig. 3, Wolleben et al., 2006; Reich, 2006).

In external galaxies and galaxy clusters, interstellar and intracluster magnetic fields can be directly traced by mapping the diffuse synchrotron emission and its polarization (Beck and Gaensler, 2004; Beck, 2005, Fig. 4). Combined with determinations of RMs for extended emission and background sources, this will allow us to derive detailed threedimensional maps of the magnetic fields in these sources. 


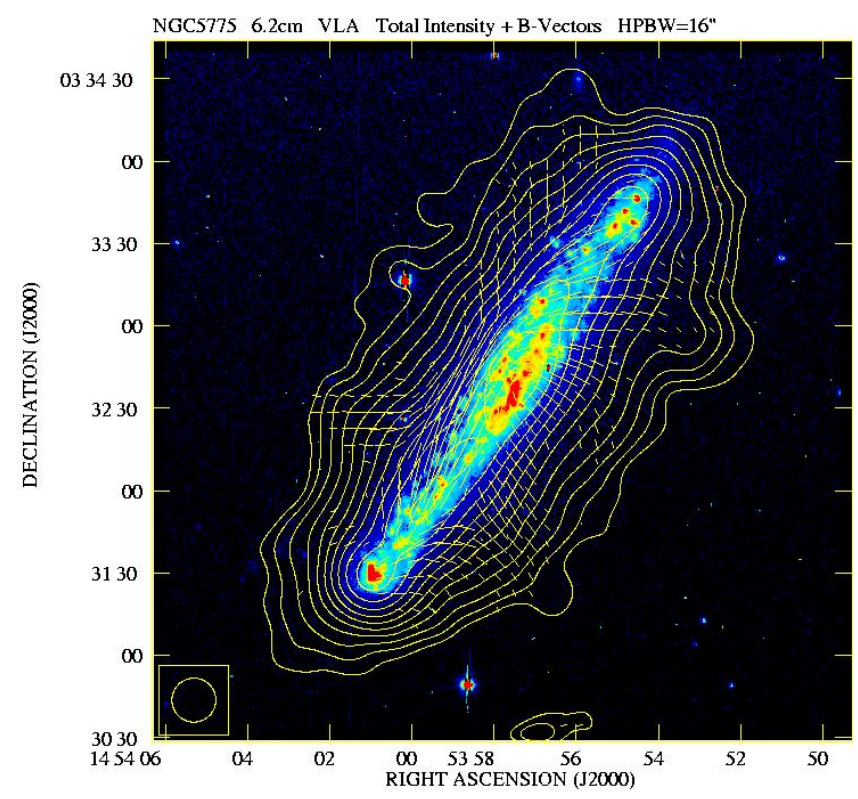

Fig. 5. Total radio emission (contours) and polarization B-vectors of the spiral galaxy NGC 5775 , observed at $4.8 \mathrm{GHz}$ with the VLA (from Tullmann et al., 2000).

SKA will provide sufficient resolution and sensitivity to identify individual features. Of special interest are the spectrum of magnetic turbulence, the number and location of magnetic reversals, the relation between magnetic and optical spiral arms, and the interaction between magnetic fields and gas flows.

LOFAR will show the extension of galaxies into the halo and the intergalactic space which emerge as a result of galactic winds or of interactions, phenomena which are observable only in a few cases with the most sensitive present-day telescopes (Figs. 5 and 6). LOFAR will also allow to trace the full extent of the magnetized halos of galaxy clusters.

\section{The origin of magnetic fields in galaxies and clusters}

The observation of large-scale patterns in RM in many galaxies (Beck, 2005) proves that some fraction of the magnetic field in galaxies has a coherent direction and hence is not generated by compression or stretching of irregular fields in gas flows. In principle, the turbulent dynamo mechanism is able to generate and preserve coherent magnetic fields, and they are of appropriate spiral shape (Beck et al., 1996). However, the physics of dynamo action is far from being understood. Primordial fields, on the other hand, are hard to preserve over a galaxy's lifetime due to diffusion, reconnection, and winding up by differential rotation. Even if they survive, they can create only specific field patterns.

The widely studied mean-field $\alpha-\Omega$ dynamo needs differential rotation and helical turbulence to operate. It gener-

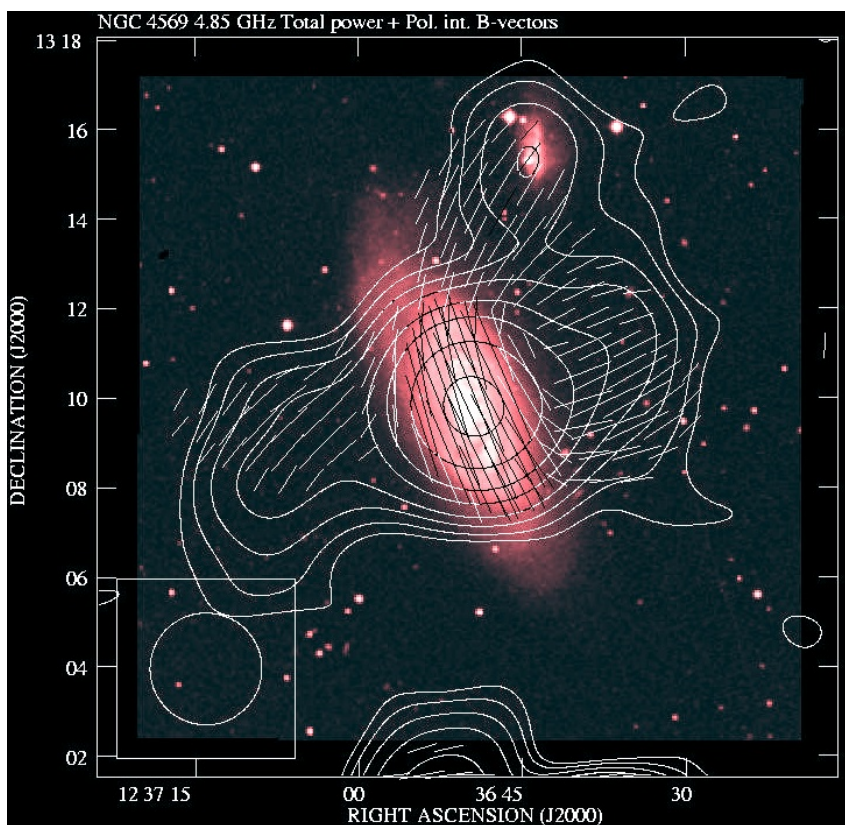

Fig. 6. Total radio emission (contours) and polarization B-vectors of the spiral galaxy NGC 4569 in the Virgo Cluster, observed at $4.8 \mathrm{GHz}$ with the Effelsberg telescope (from Chyży et al., 2006).

ates a coherent magnetic field which can be represented as a superposition of modes of different azimuthal and vertical symmetries. The existing dynamo models predict that several azimuthal modes can be excited (Beck et al., 1996), the strongest being $m=0$ (an axisymmetric spiral field), followed by the weaker $m=1$ (a bisymmetric spiral field), etc. These generate a Fourier spectrum of azimuthal RM patterns. Observations of background RMs and of diffuse polarized synchrotron emission allow direct measurements of the azimuthal modes, but these measurements are limited at present to 20 galaxies (Beck, 2000). With SKA, this sample can be increased by up to three orders of magnitude. These data can allow us to distinguish between different conditions for excitation of various dynamo modes. The presence and prevalence of reversals in the disk field structure, plus the structure of field in the halo, will together let us distinguish between dynamo and primordial models for field origin in galaxies (Beck, 2006).

Similarly, clarifying the origin of intracluster fields in galaxy clusters is a project of fundamental importance for LOFAR and SKA. Regular fields could be generated by dynamo action, cluster mergers, shock waves, or interactions between galaxies (Dolag, 2006; Shukurov et al., 2006).

\section{Magnetic fields in distant galaxies}

Measurements of magnetic fields in distant galaxies (at redshifts between $z \sim 0.1$ and $z \sim 2$ ) with LOFAR and SKA will 
provide direct information on how magnetized structures evolve and amplify as galaxies mature. The linearly polarized emission from galaxies at these distances will often be too faint to detect directly; Faraday rotation thus holds the key to studying magnetism in these sources. There are many distant, extended polarized sources (quasars and radio galaxies), providing the ideal background illumination for probing Faraday rotation in galaxies which happen to lie along the same line of sight. These experiments can deliver maps of magnetic field structures in galaxies more than 100 times more distant than discussed above.

At yet larger distances, we can take advantage of the sensitivity of the deepest SKA fields, in which we expect to detect the synchrotron emission from the youngest galaxies and proto-galaxies. Since standard dynamos need a few rotations or about $10^{9} \mathrm{yr}$ to build up a coherent galactic field (Beck et al., 1996), the detection of synchrotron emission in young galaxies would put constraints on the seed field which may call for alternative models.

\section{Intergalactic magnetic fields}

Fundamental to all the issues discussed above is the search for magnetic fields in the intergalactic medium (IGM). All of "empty" space in the Universe may be magnetized. Its role as the likely seed field for galaxies and clusters, plus the prospect that the IGM field might trace structure formation in the early Universe, places considerable importance on its discovery. A magnetic field already present at the epoch of re-ionization or even at the recombination era might have affected the processes occurring at those epochs (Subramanian, 2006). To date there has been no detection of magnetic fields in the IGM; current upper limits on the average strength of any such field suggest $\left|B_{\mathrm{IGM}}\right| \leq 10^{-8}-10^{-9} \mathrm{G}$ (Kronberg, 1994).

With LOFAR it will be possible to search for synchrotron radiation at the lowest possible levels in intergalactic space. Its detection will allow to probe the existence of magnetic fields in rarified regions of the intergalactic medium, measure their intensity, and investigate their origin and their relation to the structure formation in the early Universe. Fields of $B \simeq 10^{-9}-10^{-8} \mathrm{G}$ are expected along filaments of $10 \mathrm{Mpc}$ length with $n_{e} \simeq 10^{-5} \mathrm{~cm}^{-3}$ electron density (Kronberg, 2006) which yield Faraday rotation measures of $\mathrm{RM}=0.1-1 \mathrm{rad} \mathrm{m}^{-2}$. Their detection is a big challenge, but possible. LOFAR has a realistic chance to measure intergalactic magnetic fields for the first time.

If this all-pervading magnetic field will turn out to be even weaker, it may still be identified through the all-sky RM grid with the SKA (Sect. 5). The correlation function of the RM distribution provides the magnetic power spectrum of the IGM as a function of cosmic epoch (Blasi et al., 1999). Such measurements will allow us to develop a detailed model of the magnetic field geometry of the IGM and of the overall Universe.

Primordial fields existing already in the recombination era would induce Faraday rotation of the polarized CMB signals of the cosmic microwave background (CMB) (Kosowsky and Loeb, 1996) and generate a characteristic peak in the CMB power spectrum at small angular scales. The detection is challenging but possible with an instrument of superb sensitivity like SKA.

\section{References}

Beck, R.: Magnetic fields in normal galaxies, Phil. Trans. R. Soc. Lond. A, 358, 777-796, 2000.

Beck, R.: Magnetic fields in galaxies, in: Cosmic Magnetic Fields, edited by: Wielebinski, R. and Beck, R., Springer, Berlin, 41-68, 2005.

Beck, R.: The origin of magnetic fields in galaxies, Astr. Nachr., 327, 512-516, 2006.

Beck, R. and Gaensler, B. M.: Observations of magnetic fields in the Milky Way and in nearby galaxies with a Square Kilometre Array, in: Science with the Square Kilometre Array, edited by: Carilli, C. and Rawlings, S., New Astr. Rev., 48, 1289-1304, 2004.

Beck, R., Brandenburg, A., Moss, D., Shukurov, A., and Sokoloff, D.: Galactic magnetism: Recent developments and perspectives, Ann. Rev. Astron. Astrophys., 34, 155-206, 1996.

Blasi, P., Burles, S., and Olinto. A. V.: Cosmological magnetic field limits in an inhomogeneous Universe, Astrophys. J., 514, L79L82, 1999.

Brentjens, M. A., and de Bruyn, A. G.: Faraday rotation measure synthesis, Astron. Astrophys., 441, 1217-1228, 2005.

Brown, J. C., Taylor, A. R., and Jackel, B. J.: Rotation measures of compact sources in the Canadian Galactic Plane Survey, Astrophys. J. Suppl., 145, 213-223, 2003.

Carilli, C., and Rawlings, S.: Science with the Square Kilometre Array, New Astr. Rev., 48, 2004.

Chyży, K. T., Soida, M., Bomans, D. J., et al.: Large-scale magnetized outflows from the Virgo cluster spiral NGC 4569, Astron. Astrophys., 447, 465-472, 2006.

Dolag, K.: Simulating large-scale structure formation with magnetic fields, Astr. Nachr., 327, 575-582, 2006.

Gaensler, B. M., Beck, R., and Feretti, L.: The origin and evolution of cosmic magnetism, in: Science with the Square Kilometre Array, edited by: Carilli, C. and Rawlings, S., New Astr. Rev., 48, 1003-1012, 2004.

Han, J. L., Manchester, R. N., and Qiao, G. J.: Pulsar rotation measures and the magnetic structure of our Galaxy, Mon. Not. Royal Astr. Soc., 306, 371-380, 1999.

Han, J.L., Manchester, R. N., Lyne, A. G., Qiao, G. J., and van Straten, W.: Pulsar rotation measures and the large-scale structure of the Galactic magnetic field, Astrophys. J., 642, 868-881, 2006.

Kosowsky, A. and Loeb, A.: Faraday rotation of microwave background polarization by a primordial magnetic field, Astrophys. J., 469, 1-6, 1996.

Kronberg, P. P.: Extragalactic magnetic fields, Rep. Prog. Phys., 57, 325-382, 1994. 
Kronberg, P. P.: Extragalactic radio sources, IGM magnetic fields, and AGN-based energy flows, Astr. Nachr., 327, 517-522, 2006. Reich, W.: Galactic polarization surveys, astro-ph/0603465, 2006.

Reich, W., Fürst, E., Reich, P., Uyanıker, B., Wielebinski, R., and Wolleben, M.: The Effelsberg 1.4 GHz medium Galactic latitude survey (EMLS), in: The Magnetized Interstellar Medium, edited by: Uyanıker et al., Copernicus, Katlenburg, p. 45, 2004.

Shukurov, A., Subramanian, K., and Haugen, N. E. L.: The origin and evolution of cluster magnetism, Astr. Nachr., 327, 583-586, 2006.

Sokoloff, D. D., Bykov, A. A., Shukurov, A., Berkhuijsen, E. M., Beck, R., and Poezd, A. D.,: Depolarization and Faraday effects in galaxies, Mon. Not. Royal Astr. Soc., 299, 189-206, 1998, and Mon. Not. Royal Astr. Soc., 303, 207-208, 1999 (Erratum).
Stepanov, R., Frick, P., Shukurov, A., and Sokoloff, D.: Wavelet tomography of the Galactic magnetic field, Astron. Astrophys., 391, 361-368, 2002.

Subramanian, K.: Primordial magnetic fields and CMB anisotropies, Astr. Nachr., 327, 403-409, 2006.

Tüllmann, R., Dettmar, R.-J., Soida, M., Urbanik, M., and Rossa, J.: The thermal and non-thermal gaseous halo of NGC 5775, Astron. Astrophys., 364, L36-L41, 2000.

Wolleben, M., Landecker, T.L., Reich, W., and Wielebinski, R., An absolutely calibrated survey of polarized emission from the northern sky at $1.4 \mathrm{GHz}$, Astron. Astrophys., 448, 411-424, 2006. 